\title{
Abundance analysis of a CEMP-no star in the Carina dwarf spheroidal galaxy $\star, \star \star$
}

\author{
A. Susmitha ${ }^{1,2}$, A. Koch $^{3}$, and T. Sivarani ${ }^{1}$ \\ 1 Indian Institute of Astrophysics, Bangalore-34, India \\ e-mail: [susmitha;sivarani]@iiap.res.in \\ 2 Indian Institute of Science, Bangalore-12, India \\ 3 Department of Physics, Lancaster University, Lancaster, LA1, 4YB, UK \\ e-mail: a.koch1@lancaster.ac.uk
}

Received 13 March 2017 / Accepted 15 May 2017

\begin{abstract}
Carbon-enhanced metal-poor (CEMP) stars bear important imprints of the early chemical enrichment of any stellar system. While these stars are known to exist in copious amounts in the Milky Way halo, detailed chemical abundance data from the faint dwarf spheroidal (dSph) satellites are still sparse, although the relative fraction of these stars increases with decreasing metallicity. Here, we report the abundance analysis of a metal-poor $([\mathrm{Fe} / \mathrm{H}]=-2.5 \mathrm{dex})$, carbon-rich $([\mathrm{C} / \mathrm{Fe}]=1.4 \mathrm{dex})$ star, ALW-8, in the Carina dSph using high-resolution spectroscopy obtained with the ESO/UVES instrument. Its spectrum does not indicate any over-enhancements of neutron capture elements. Thus classified as a CEMP-no star, this is the first detection of this kind of star in Carina. Another of our sample stars, ALW-1, is shown to be a CEMP- $s$ star, but its immediate binarity prompted us to discard it from a detailed analysis. The majority of the 18 chemical elements we measured are typical of Carina's field star population and also agree with CEMP stars in other dSph galaxies. Similar to the only known CEMP-no star in the Sculptor dSph and the weak-r-process star HD 122563 , the lack of any strong barium-enhancement is accompanied by a moderate overabundance in yttrium, indicating a weak $r$-process activity. The overall abundance pattern confirms that, also in Carina, the formation site for CEMP-no stars has been affected by both faint supernovae and by standard core collapse supernovae. Whichever process was responsible for the heavy element production in ALW-8 must be a ubiquitous source to pollute the CEMP-no stars, acting independently of the environment such as in the Galactic halo or in dSphs.
\end{abstract}

Key words. stars: abundances - binaries: spectroscopic - stars: carbon - Galaxy: abundances - galaxies: dwarf

\section{Introduction}

Observational studies of metal-poor stars in the Milky Way (MW) and other nearby galaxies reveal that the early Universe has experienced various nucleosynthetic mechanisms. The diverse chemical composition these stars preserve in their photosphere is the result of such nucleosynthetic mechanisms and detailed studies of their abundances yield information about the nature of their progenitors and how the latter contributed to the interstellar medium (ISM) through various (explosive) mechanisms. The diversity in the stellar chemical properties becomes more important and unique as the metallicity of the stars decreases.

Metal-poor stars in the MW have been extensively studied and classified into different groups based on their metallicity, with further distinctions based on the abundance of certain key elements (e.g., Beers \& Christlieb 2005; Aoki et al. 2007). Amongst these, Carbon-enhanced metal-poor (CEMP) stars (defined by $[\mathrm{Fe} / \mathrm{H}]<-2.0$ and $[\mathrm{C} / \mathrm{Fe}]>0.7)$ are of great interest since the origin of the carbon overabundance is versatile and closely related to the formation and enrichment history of

\footnotetext{
$\star$ Based on observations collected at the European Southern Observatory at Paranal, Chile; Large Programme proposal 171.B- 0520.

$\star \star$ Table A.1 is also available at the CDS via anonymous ftp to cdsarc.u-strasbg. fr (130.79.128.5) or via

http://cdsarc.u-strasbg.fr/viz-bin/qcat?J/A+A/606/A112
}

the host stellar system (Aoki et al. 2007; Masseron et al. 2010; Carollo et al. 2014; Bonifacio et al. 2015; Hansen et al. 2016a; Koch et al. 2016; Lee et al. 2017). These stars are primarily categorized based on the presence and absence of neutron capture elements as CEMP- $s$, CEMP- $r$, or CEMP- $r / s$, versus CEMPno, respectively. Amongst these sub classes, CEMP- $s$ stars with their strong enhancements in $s$-process elements are mainly found to be members of a binary system (Aoki et al. 2007; Starkenburg et al. 2014; Hansen et al. 2016c). This favors the idea that their carbon-enhancement is due to mass transfer from a companion, which was once in its Asymptotic Giant Branch (AGB) phase and has since faded into a white dwarf. Thus, the carbon- and $s$-process-rich material had been accreted onto the surface of the presently observed star, which does not necessarily reflect the properties of the genuine ISM from which it was born.

CEMP-no stars, on the other hand, are often found not to be associated with binaries (according to Hansen et al. 2016b, 4 out of 24 stars in the radial velocity monitoring show binarity) and, as such, their abundance patterns are those imprinted by the ISM. Hansen et al. (2016b) stated that CEMPno stars are bona fide second generation stars, bearing the primordial remnants of enrichment from an early generation of stars. The frequency of CEMP-no stars increases with decreasing metallicity, and below an $[\mathrm{Fe} / \mathrm{H}]$ of approximately -3.5 dex almost all C-rich stars are of the CEMP-no class 
(Bonifacio et al. 2015; Hansen et al. 2016a). The abundance pattern observed in CEMP-no stars matches well with models of primordial faint Supernovae (SNe) that experienced mixing and fall-back, and models of zero-metallicity-spin stars having high rotational velocity (e.g., Umeda \& Nomoto 2003; Meynet et al. 2006; Kobayashi et al. 2011; Ishigaki et al. 2014). In this regard, Yoon et al. (2016) and Hansen et al. (2016a) suggest that probably more than one class of first-generation progenitors is required to account for the abundance patterns of CEMP-no stars.

Recent studies have uncovered several carbon-rich stars in dwarf spheroidals (dSph) and ultrafaint dwarf satellites around the MW (Frebel et al. 2010b; Lai et al. 2011; Norris et al. 2010a; Ji et al. 2016a). Broadly consistent with the properties of CEMP stars in the Galactic halo, these objects were found across the entire metallicity range down to $[\mathrm{Fe} / \mathrm{H}] \sim-3.5$ dex and as metal rich as -2.0 dex and above, with varying $\mathrm{C}$-enhancements from moderate values of $[\mathrm{C} / \mathrm{Fe}] \sim 0.5$ up to $2.3 \mathrm{dex}$, and drawn from the CEMP-no and CEMP- $s$ subclasses. Moreover, these stars have now been found and analyzed in detail in the more luminous dSphs such as Sculptor and Sextans (Honda et al. 2011; Skúladóttir et al. 2015; Salgado et al. 2016). Here, the latter works stand out in that they provide the first carbon-rich stars within the metal-rich tails of the galaxies' metallicity distributions $($ at $[\mathrm{Fe} / \mathrm{H}]=-2$ and $-1 \mathrm{dex}$ ). Even though all of the more luminous dSphs contain old stellar populations (Grebel 1997), none of them appear to show any CEMP population in the extremely metal-poor regime below -2 dex, owing to the shift in the metallicity distribution function towards the metalrich side with increasing galaxy luminosity (Kirby et al. 2011; Salvadori et al. 2015).

Amongst the classical dSphs, Carina is of special interest because of its unusual, episodic star formation history, where each of the stellar populations has also experienced distinct chemical enrichment (Mould \& Aaronson 1983; Smecker-Hane et al. 1994; Monelli et al. 2003; Tolstoy et al. 2003; Koch et al. 2008). Azzopardi et al. (1986, herafter ALW) detected ten carbon-rich (CH- and C-) stars in Carina. Two of those (ALW-6 and ALW-7) were analyzed by Abia et al. (2008), with a particular focus on the origin of the $s$-process elements in these stars. At $[\mathrm{Fe} / \mathrm{H}]=$ -1.8 to -2 dex and $[\mathrm{Ba} / \mathrm{Fe}]>1.9$ dex, both qualify as CEMP$s$ stars, where mass transfer of processed material acted as the source of the abundance enhancements. Conversely, no CEMPno stars have been identified in Carina to date.

In this paper, we report on the abundance analysis of the first CEMP-no star in Carina (ALW-8), which shows no enhancement in neutron capture elements. Another member of the ALWsample (ALW-1) could be shown to be a CEMP-s star; however its binarity inhibited a detailed abundance analysis. This paper is organized as follows: in Sect. 2, we briefly recapitulate the observations and data for the stars, and in Sect. 3 we describe in detail our abundance analysis for ALW-8, the results of which are presented in Sect. 4. In Sect. 5, the resulting abundances are discussed in terms of the origin of the elements in this star, before we conclude our measurements in Sect. 6.

\section{Observations and data reduction}

The data were obtained in the course of the ESO Large Programme 171.B-0520 (PI: G. F. Gilmore) that aimed at studying the kinematic and chemical characteristics of Local Group dSphs (Koch et al. 2006, 2008). Along with high-resolution $(R \sim 40000)$ spectra of ten red giants that were observed with the Ultraviolet and Visual Echelle Spectrograph (UVES) at the ESO/VLT in multi-object mode, two of the targets turned out to

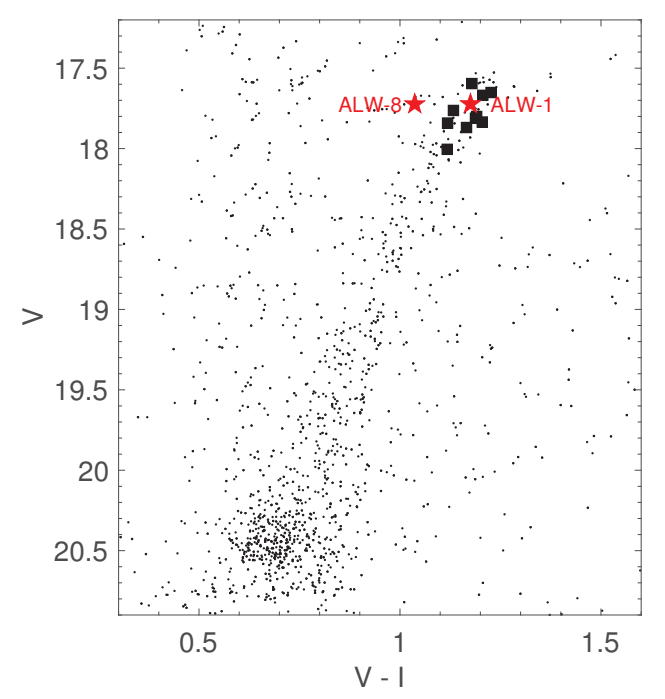

Fig. 1. Upper color magnitude diagram of Carina's central 10", using photometry from the ESO Imaging Survey. Stars shown as black squares are the red giant targets of Koch et al. (2008), while the filled red star symbols indicate the carbon stars from the present work.

Table 1. Photometric, kinematic, and atmospheric parameters of the target stars.

\begin{tabular}{cccc}
\hline \hline Property & ALW-1 & ALW-8 & Reference $^{a}$ \\
\hline$\alpha(\mathrm{J} 2000.0)$ & $06: 41: 08.58$ & $06: 41: 46.27$ & 1 \\
$\delta(\mathrm{J} 2000.0)$ & $-50: 47: 50.1$ & $-50: 58: 55.9$ & 1 \\
$V$ & 17.68 & 17.88 & 2 \\
$I$ & 16.33 & 16.41 & 2 \\
$J$ & 15.235 & 15.465 & 3 \\
$H$ & 14.557 & 14.778 & 3 \\
$K$ & 14.295 & 14.509 & 3 \\
$E(B-V)$ & 0.053 & 0.064 & 4 \\
$v_{\mathrm{HC}}\left[\mathrm{km} \mathrm{s}{ }^{-1}\right]$ & $226.2 / 256.2^{b}$ & 223.3 & 5 \\
$T_{\mathrm{eff}}[\mathrm{K}]$ & $\ldots$ & 4150 & 5 \\
$\log g$ & $\ldots$ & 1.00 & 5 \\
$\xi\left[\mathrm{km} \mathrm{s}{ }^{-1}\right]$ & $\ldots$ & 2.3 & 5 \\
{$[\mathrm{Fe} / \mathrm{H}]$} & $\sim-2.8$ & -2.5 & 5 \\
$\mathrm{C} / \mathrm{O}$ & $\ldots$ & 1.5 & 5 \\
${ }^{12} \mathrm{C} /{ }^{13} \mathrm{C}$ & $\ldots$ & 9 & 5 \\
\hline
\end{tabular}

Notes. ${ }^{(a)}$ References: 1: ALW; 2: Walker et al. (2009); 3: 2MASS (Cutri et al. 2003); 4: Schlegel et al. (1998); 5: this work. ${ }^{(b)}$ Radial velocity from the two epochs of our observations.

be carbon-rich stars as was obvious from their strong molecular bands. Coincidentally, these were part of the ALW sample, namely ALW-1 and ALW-8. The C-rich nature of ALW-8 was also noted by Venn et al. (2012), but discarded from their further analysis. Our observations and data reduction for these two stars are identical to those of the red giant sample of Koch et al. (2008), to which we refer the reader for details. The resulting UVES spectra cover a full wavelength range from 4800 to $6800 \AA$ A.

Figure 1 shows the location of the stars in a color magnitude diagram with photometry taken from the ESO Imaging Survey (Nonino et al. 1999), and their general properties are listed in Table 1. 


\subsection{Radial velocity and binarity}

In order to correct for the shift in spectral lines, the spectra of both stars were cross-correlated against a synthetic spectrum generated on the initial assumptions of temperature, surface gravity, and the mean metallicity of Carina (Koch et al. 2006). The resulting heliocentric velocity of ALW-8 of $223.3 \pm$ $1.0 \mathrm{~km} \mathrm{~s}^{-1}$ is not only in excellent agreement with Carina's systemic velocity, but also agree well with the values of these stars found by Walker et al. (2009).

For ALW-1, however, the two epochs of our observations returned velocities deviating by $30 \mathrm{~km} \mathrm{~s}^{-1}$. Likewise, the data of Walker et al. (2009, and priv. comm.) and Hansen (priv. comm.) indicate significant radial velocity variations that suggest that ALW-1 is in fact part of a binary system. Therefore, we discarded the spectrum from further consideration due to the large uncertainties imposed by its low signal-to-noise ratio and difficulties in continuum placement due to veiling (e.g., Thompson et al. 2008). A cursory analysis indicated a metallicity of $-2.8 \mathrm{dex}$, a large $[\mathrm{C} / \mathrm{Fe}]$ ratio of $\sim 1.4 \mathrm{dex}$, and a strong enhancement in $s$-process elements with a $[\mathrm{Ba} / \mathrm{Fe}]$ ratio of approximately 2 dex. This shows that ALW-1 is likely to be a CEMP- $s$ star.

\section{Abundance analysis}

Throughout our work, we used the spectral synthesis code TURBOSPECTRUM developed by Plez (2012). To this end, we interpolated the final stellar atmospheric model from a grid of model photospheres (Mészáros et al. 2012) in which the ATLAS9 and MARCS codes were modified with an updated $\mathrm{H}_{2} \mathrm{O}$ linelist and with a wide range of carbon- and $\alpha$-element enhancements. Local thermodynamic equilibrium (LTE) has been assumed for all species.

We adopted the Solar abundances from Asplund et al. (2009) and Solar isotopic ratios were used for all the elements unless noted otherwise (see Sect. 4.3.). Our line lists for atomic lines were assembled from the VALD database Kupka et al. (1999) and details are given in Table 2. Hyperfine structure (HFS) has been accounted for for $\mathrm{Li}, \mathrm{Sc}, \mathrm{Ba}$, and $\mathrm{Eu}$, although the corrections were negligible in the latter two cases. Finally, for the relevant molecules we employed the $\mathrm{CH}$ line list compiled by $\mathrm{T}$. Masseron (priv. comm.) and CN data from Plez \& Cohen (2005).

\subsection{Equivalent-width measurements}

The spectrum of the CEMP star ALW-8 shows a wealth of molecular features that contaminate the atomic lines. This severely hindered the measurements of precise equivalent widths (EWs) for a large number of lines. To this end, we constrained our analysis to such few lines that are devoid of contributions from molecular bands. In order to identify such unblended lines, we synthesized the full spectral range using the representative carbon, nitrogen, and oxygen abundances, and checked for those lines that remained free from molecular features. In practice, EWs were measured by fitting Gaussian profiles to the features using IRAF's splot task. The uncertainties in the measurements were determined via the revised Cayrel formula (Cayrel de Strobel \& Spite 1988; Battaglia et al. 2008). The final linelist is given in Table A.1, which is also available at the CDS.
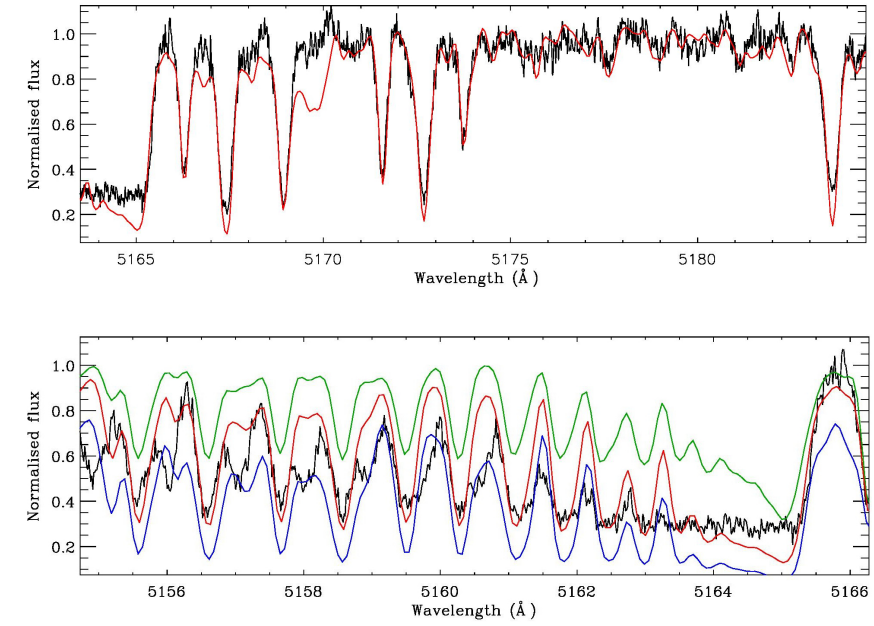

Fig. 2. Top panel: region of the Mg I triplet in ALW-8 (black line). The best-fit synthetic spectrum is plotted in red. The additional feature at $5170 \AA$ is due to the chosen carbon abundance. Bottom panel: synthesis of the $\mathrm{C}_{2}$-band in ALW-8. The best-fit spectrum is shown in red $([\mathrm{C} / \mathrm{Fe}]=+1.39)$, while the blue and green lines have been computed for carbon abundances that differ by \pm 0.2 dex.

\subsection{Stellar parameters}

The effective temperature of ALW-8 has been estimated using various color indices from the literature (Table 1), and using the color-transformation of Alonso et al. (1999). To this end, we adopted an initial metallicity of $-2.5 \mathrm{dex}$ and a reddening of $E(B-V)=0.064$ (Schlegel et al. 1998). At $T_{\text {eff }}=(4150 \pm 40 \mathrm{~K}$, $4200 \pm 170 \mathrm{~K}, 4240 \pm 125 \mathrm{~K})$ from the $V-K, J-H$, and $J-K$ colors, respectively, all photometric values are in very good agreement within the errors. In the following, we adopt the value from the $V-K$ color of $4150 \pm 40 \mathrm{~K}$ as the stellar temperature. Given the broad color range spanned by this index, this is the most reliable indicator, and it also corresponds to the error-weighted mean of all values above. This also yielded a flat trend of abundance of Fe I lines with excitation potential. By demanding that the slope of the iron abundances from the neutral lines with excitation potential do not change by more than one standard deviation of the Fe I line abundances, we placed an error of $100 \mathrm{~K}$ on the spectroscopic temperature.

The surface gravity, $\log g$ was derived by fitting a grid of synthetic spectra to the $\mathrm{Mg}$ triplet lines around $5170 \AA$, where we adopted the temperature derived above as the model's $T_{\text {eff }}$. The best-fit model thus found is indicated in Fig. 2. Acceptable fits, in particular accounting for the Mg triplet's wings, were still obtained within variations of $\pm 0.25 \mathrm{dex}$, which we adopted as the error on our $\log g$ determination. Finally, the microturbulent velocity, $\xi$, was set by removing any trend of the abundance from the Fe I lines with the reduced width, $\log (E W / \lambda)$. The resulting value was found to be $\xi=2.3 \mathrm{~km} \mathrm{~s}^{-1}$ with an error of $0.2 \mathrm{~km} \mathrm{~s}^{-1}$, as determined by the point where the Fe I abundance did not change by more than $1 \sigma$. While fixing the surface gravity, the $\mathrm{C}$-abundance was iteratively changed from $\log \epsilon(\mathrm{C})=6.42$ to $\log \epsilon(\mathrm{C})=8.02$ in steps of 0.2 dex to converge on the best-fit parameters. All stellar parameters obtained in this way are listed in Table 1.

\section{Abundance results}

All abundance results are listed in Table 2, together with our adopted Solar values from Asplund et al. (2009). In Fig. 3, 
Table 2. Abundance results.

\begin{tabular}{ccccccc}
\hline \hline Species & $\log \epsilon_{\odot}$ & $\log \epsilon$ & {$[\mathrm{X} / \mathrm{Fe}]$} & $\sigma_{\log \epsilon}$ & $N$ & $\sigma_{\text {sys }}^{\text {tot }}$ \\
\hline $\mathrm{Li}$ I & 1.05 & 0.00 & 1.45 & 0.30 & syn & 0.32 \\
$\mathrm{C}\left(\mathrm{C}_{2}\right)$ & 8.43 & 7.32 & 1.39 & 0.20 & syn & 0.15 \\
$\mathrm{~N}(\mathrm{CN})$ & 7.83 & 6.92 & 1.59 & 0.30 & syn & 0.14 \\
$\mathrm{O}$ I & 8.69 & 7.08 & 0.89 & 0.30 & syn & 0.10 \\
$\mathrm{Na}$ I & 6.24 & $4.62_{\mathrm{NLTE}}$ & $0.88_{\text {NLTE }}$ & 0.12 & 2 & 0.09 \\
$\mathrm{Mg}$ I & 7.60 & 5.40 & 0.30 & $\ldots$ & 1 & 0.13 \\
$\mathrm{Ca}$ I & 6.34 & 4.26 & 0.42 & 0.27 & 5 & 0.13 \\
$\mathrm{Sc} \mathrm{II}$ & 3.15 & 1.17 & 0.52 & 0.09 & 3 & 0.16 \\
$\mathrm{Ti}$ I & 4.95 & 2.75 & 0.30 & 0.37 & 7 & 0.18 \\
$\mathrm{Ti}$ II & 4.95 & 2.85 & 0.40 & 0.15 & 3 & 0.16 \\
$\mathrm{Cr}$ I & 5.64 & 2.94 & -0.20 & 0.29 & 5 & 0.16 \\
$\mathrm{Mn}$ I & 5.43 & 2.63 & -0.30 & 0.10 & 2 & 0.19 \\
$\mathrm{Fe}$ I & 7.50 & 5.00 & 0.00 & 0.12 & 11 & 0.12 \\
$\mathrm{Fe}$ II & 7.50 & 4.99 & 0.01 & 0.10 & 2 & 0.19 \\
$\mathrm{Ni}$ I & 6.22 & 3.56 & -0.16 & 0.15 & 3 & 0.11 \\
$\mathrm{Zn} \mathrm{I}$ & 4.56 & 2.24 & 0.18 & $\ldots$ & 1 & 0.12 \\
$\mathrm{Y} \mathrm{II}$ & 2.21 & 0.00 & 0.29 & $\ldots$ & syn & 0.11 \\
$\mathrm{Zr}$ II & 2.58 & $<0.50$ & $<0.42$ & $\ldots$ & syn & 0.42 \\
$\mathrm{Ba}$ II & 2.18 & -0.86 & -0.55 & $\ldots$ & syn & 0.19 \\
$\mathrm{Eu}$ I & 0.52 & $<-1.50$ & $<0.48$ & $\ldots$ & syn & 0.86 \\
\hline
\end{tabular}

our results are compared to literature measurements in the Galactic disks (Koch \& Edvardsson 2002; Bensby et al. 2014; Battistini \& Bensby 2016) and halo (Roederer et al. 2014), where we also included data for C-normal stars in Carina (Koch et al. 2008; Venn et al. 2012; Fabrizio et al. 2015), other C-rich stars in MW dSph satellites, including Carina's ALW-6 and -7 (Abia et al. 2008), and the sparse available data from a few ultrafaint galaxies (Geisler et al. 2005; Frebel et al. 2010b; Norris et al. 2010b; Honda et al. 2011; Skúladóttir et al. 2015; Salgado et al. 2016; Ji et al. 2016a,b). We note that the Galactic data have not been preselected to show exclusively CEMP stars, but they are, rather, representative of the metal-poor MW field population. Similarly, for the faint dSphs, we only show those stars that have several key elements derived from highresolution spectra, while the overall detection rate of carbon excesses from low-resolution spectra without further chemical follow-up is larger (e.g., Lai et al. 2011). Moreover, a few of the CEMP-stars in the faintest dSphs often fall within the CEMP category by a margin of their [C/Fe] ratio (Aoki et al. 2007).

\subsection{Abundance errors}

In order to quantify the statistical error due to contributions from uncertainties in EWs and atomic parameters, we list in Table 2 the $1 \sigma$ line-to-line scatter, $\sigma_{\log \epsilon}$ and the number of lines on which each element's abundance was based. For those few cases, where the abundance could only be derived from less than three lines, this random error was adopted as 0.10 as an empirical, conservative upper limit. For the abundances derived via spectrum synthesis, the quoted abundance uncertainty was determined via the goodness of the least-squares fit.

Systematic errors in our abundances were derived by computing new models, where each stellar parameter $\left(T_{\text {eff }}, \log g, \xi\right)$ was varied by a fixed amount $\left( \pm 100 \mathrm{~K}, \pm 0.25 \mathrm{dex}, \pm 0.2 \mathrm{~km} \mathrm{~s}^{-1}\right)$, and from those, new abundances were computed in an identical manner as before. The resulting line-to-line change upon these parameter variations is listed in Table A.1, and the combined effect was determined by adding the contributions in quadrature. These systematic uncertainties are listed in the last column of Table 2 .

\subsection{Iron}

Many iron lines are severely blended with $\mathrm{CN}$ and $\mathrm{C}_{2}$ molecular features, but we were able to measure the EWs of $11 \mathrm{Fe} I$ lines that are free of blends. In addition, we synthesized the full spectral range in its entirety in order to assess the accuracy of the abundance analysis and the influence of such blending. As a result, the values from the EW analysis and spectral synthesis are in excellent agreement. We thus report the Fe-abundance of ALW-8 as $[\mathrm{Fe} / \mathrm{H}]=-2.50 \pm 0.04$.

The covered spectral range also allowed us to determine the abundance from two unblended Fe II lines. The resulting abundance from these lines indicates an excellent ionization equilibrium in that $[\mathrm{Fe} \mathrm{I} / \mathrm{II}]=0.01 \pm 0.13$, which also renders our surface gravities from the synthetic grid fitting reliable.

\subsection{Carbon, nitrogen, oxygen, and ${ }^{12} \mathrm{C} /{ }^{13} \mathrm{C}$ ratio}

The carbon abundance of this star has been derived by fitting the $\mathrm{C}_{2}$ molecular band heads at $5164 \AA$ and $5635 \AA$ (Fig. 2). Both features yielded the same abundance of $\log \epsilon(\mathrm{C})=7.3 \pm 0.2 \mathrm{dex}$. The isotopic ratio ${ }^{12} \mathrm{C} /{ }^{13} \mathrm{C}$ was derived by fitting the ${ }^{13} \mathrm{C}$ contribution to the band at $5634 \AA$, resulting in a ratio of 9 , which is consistent with other CEMP-no stars (Sivarani et al. 2006; Aoki et al. 2007). The equilibrium ratio for CNO-cycled material is $\sim 4$ and the value in ALW-8, resulting from the production of ${ }^{13} \mathrm{C}$ in the $\mathrm{CN}$ cycle, indicates a high level of processing, in line with the evolved nature of this star (Charbonnel et al. 1998; Gratton et al. 2000). Since the strong CN band at $4215 \AA$ is not covered by our spectrum, we used $\mathrm{CN}$ lines in the wavelength range from $5635-6700 \AA$ by iteratively changing the nitrogen abundance of the synthetic spectra by 0.2 dex and fitting this entire spectral region. This yielded a best-fit value of $[\mathrm{N} / \mathrm{Fe}]=1.6$.

Finally, the oxygen abundance of ALW-8 was derived by using the [O I] lines at 6300, $6363 \AA$ (Fig. 4). Since these lines are blended with $\mathrm{CN}$ features, a slight change in the nitrogen abundance affects the derived oxygen abundance. Thus, the carbon abundance was first fixed to be the value obtained above from the $C_{2}$ band, after which the oxygen and nitrogen abundances were iteratively adjusted to fit the [O I] lines, without affecting other $\mathrm{CN}$ features. The resulting $\mathrm{O}$-abundance from both lines differs by 0.3 dex owing to the iterative process involving both $\mathrm{N}$ and $\mathrm{O}$, and we adopt this difference as our final uncertainty on the reported nitrogen and oxygen abundances.

\subsection{Lithium}

The Li-abundance for the star was determined by synthesizing the resonance doublet at $6707 \AA$ which yielded $A(\mathrm{Li})=0.0$. Due to the presence of prominent $\mathrm{CN}$ bands in this region, affecting the continuum placement, we assigned a fitting uncertainty of 0.3 dex to this result. The low value for $A(\mathrm{Li})$ we found is consistent with the star being an evolved giant, in which Li is easily destroyed at the high interior temperatures that it is exposed to due to the convective mixing of material (e.g., Gratton et al. 2000; Lind et al. 2009; Skúladóttir et al. 2015). In contrast, unevolved CEMP stars show Li-abundances that are lower than the standard plateau value of 2.2 dex (Spite \& Spite 1982; Sbordone et al. 2010; Masseron et al. 2012; Bonifacio et al. 2015). 


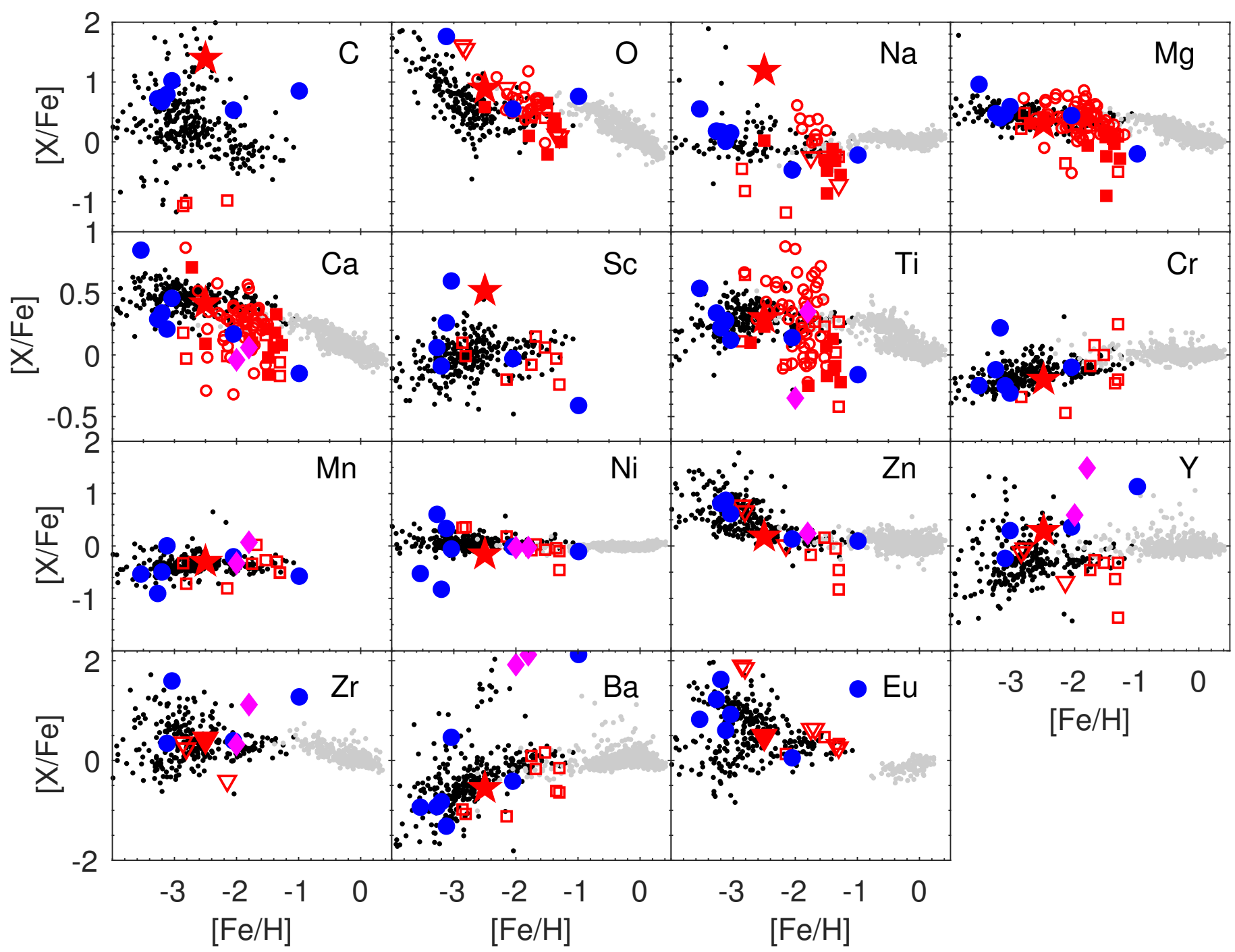

Fig. 3. Abundance ratios for ALW-8 (red star symbol and, for the case of upper limits, filled red triangles) in comparison with MW and dSph samples from the literature. Data are from Roederer et al. (2014) for halo stars (black dots); Bensby et al. (2014) for disk stars (gray points), except for $\mathrm{Zr}$ (Battistini \& Bensby 2016) and Eu (Koch \& Edvardsson 2002). Carina field star abundances were taken from Koch et al. (2008, filled red squares); Venn et al. (2012, open red squares); and Fabrizio et al. (2015, open red circles), while open red triangles indicate upper limits from these studies. Blue points designate C-rich stars in luminous and ultrafaint dSphs (Geisler et al. 2005; Frebel et al. 2010b; Norris et al. 2010b; Honda et al. 2011; Skúladóttir et al. 2015; Salgado et al. 2016; Ji et al. 2016a,b). Finally, the CEMP-s stars in Carina from Abia et al. (2008) are shown as magenta diamonds.

\section{5. $\alpha$-elements: $\mathrm{Mg}, \mathrm{Ca}$, and Ti}

Clean lines of $\mathrm{Mg}, \mathrm{Ca}$, and $\mathrm{Ti}$ are available in the spectrum of ALW-8 and the abundances of the respective elements have been determined from their EWs. The Mg I triplet lines at $5172 \AA$ and $5183 \AA$ are very strong (at $E W s>300 \mathrm{~m} \AA$ ) and saturated; conversely, the line at $5711 \AA$ is strongly blended with molecular features and thus unusable for an abundance determination. Hence, we base our measurement of the $\mathrm{Mg}$-abundance on the unblended line at $5528 \AA$ at moderate strength $(120 \mathrm{~m} \AA)$, resulting in a $[\mathrm{Mg} / \mathrm{Fe}]$ ratio of $0.3 \mathrm{dex}$.

A poor mixing has been found between Carina's old and intermediate-age populations in terms of their $\mathrm{Mg}$ abundances (Shetrone et al. 2003; Koch et al. 2008; Lemasle et al. 2012; Venn et al. 2012). Here, older stars with systematically lower $\mathrm{Fe}$ abundances show depleted $[\mathrm{Mg} / \mathrm{Fe}]$ ratios, whereas the intermediate-age population is more strongly enhanced in $\mathrm{Fe}$ and the $\alpha$-element Mg. This left Venn et al. (2012) to conclude that the second phase of star formation in Carina occurred out of gas that was already pre-enriched in the $\alpha$-elements. Moreover, the gas out of which the older stars formed was inhomogeneously mixed itself, leading to a broad spread in the $[\mathrm{Mg} / \mathrm{Fe}]$ ratios at low metallicities. At its low $[\mathrm{Fe} / \mathrm{H}]$ and the elevated $[\mathrm{Mg} / \mathrm{Fe}]$, ALW-8 is clearly part of Carina's old population.

Five $\mathrm{Ca}$ I lines have been used to determine a halo-like $[\mathrm{Ca} / \mathrm{Fe}]$ ratio of $0.42 \mathrm{dex}$, while, for $\mathrm{Ti}$, eight and three neutral and ionized lines were clean and detectable, respectively. These indicate that ionization balance is matched very well within the uncertainties, at $[\mathrm{Ti} \mathrm{I} / \mathrm{II}]=0.10 \pm 0.18 \mathrm{dex}$. No Si lines are present in the available range of the spectrum.

The straight average of the three $\alpha$-elements amounts to an enhancement of $[\alpha / \mathrm{Fe}]=0.36 \mathrm{dex}$. Even though the trends indicating the poor mixing between the components is less pronounced in the other $\alpha$-abundances, there is still considerable spread in $\mathrm{Ca}$ and in particular Ti (Fabrizio et al. 2015). ALW-8 behaves rather halo-like in its enhancements. While this overlap is also seen for several of the CEMP stars in other dSphs, two of these stand out clearly. One is the most metal-poor of these 

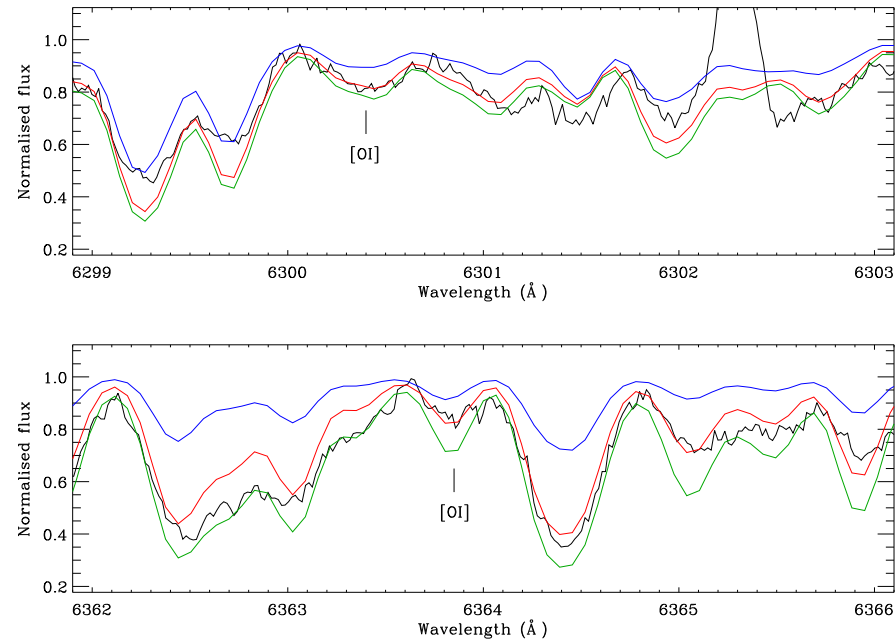

Fig. 4. Regions around the $[\mathrm{O} \mathrm{I}]$ lines in ALW-8. The best spectrum (red) was computed with $\log \epsilon(\mathrm{O})=6.93$ (top panel) and $\log \epsilon(\mathrm{O})=7.23$ (bottom panel), respectively. The green and blue lines indicate syntheses corresponding to the range of uncertainty of \pm 0.3 dex in oxygen abundance. The poor fitting of $\mathrm{CN}$ features around [OI] comes solely from the change in the oxygen abundance.

objects, (the CEMP-no star in Segue 1; Norris et al. 2010b), which is $\alpha$-enhanced to almost one dex. Secondly, the most metal-rich C-rich star in Sculptor, at -1 dex (Geisler et al. 2005), shows subsolar $[\alpha / \mathrm{Fe}]$ ratios; although we note that it represents the typical $[\alpha / \mathrm{Fe}]$ ratio of stars at similar metallicity in Sculptor. Also the CEMP- $s$ stars ALW-6 and -7 are towards the low- $\alpha$ tail of Carina's distribution. The fact that ALW-8 and the majority of the other C-stars follow the overall trends in the $\alpha$-elements very well is not surprising, since the nucleosynthesis of the $\alpha$ elements is decoupled from the channels responsible for the C-overabundances.

\subsection{Odd-Z elements: $\mathrm{Na}$ and Sc}

Since the Na D lines are too strong $(E W \sim 300 \mathrm{~m} \AA)$ for a meaningful abundance analysis, we relied on the weaker absorption lines at 5682 and $5688 \AA$. We have incorporated NLTE corrections from Lind et al. (2011) and the resulting NLTE [Na/Fe] ratio is high, at 0.9 dex, skimming the upper distribution of halo stars and Carina stars. Fabrizio et al. (2015) found that a correlation between $\mathrm{Na}$ and $\mathrm{O}$ in their Carina stars matched well with that in MW halo field stars, supporting the similarity between the chemical enrichment history of the MW halo and Carina stars (Idiart \& Thévenin 2000; Geisler et al. 2007). Unfortunately, no $\mathrm{Na}$ abundances were derived for the old stars in their sample. While, at first glance, the correlation noted by Fabrizio et al (2015) appears to be driven by a single, low-Na, low-O star, the very high $\mathrm{Na}$ and $\mathrm{O}$ abundances in $\mathrm{ALW}-8$ provide a unique match to this trend at the other extreme, bolstering the presence of this correlation beyond the intermediate-age population. This suggests that, apart from an O-enhancement in the CNO processing that this star experienced, the gas out of which it formed was already strongly pre-enriched in $\mathrm{O}$ and $\mathrm{Na}$ via previous Type-II $\mathrm{SNe}$ events.

No lines of $\mathrm{Al}$ and $\mathrm{K}$ could be measured in the spectrum and all available V-lines are too heavily blended with molecular features to infer any abundance.
The Sc-abundance we measured from three generally strong and unblended lines yields a high $[\mathrm{Sc} / \mathrm{Fe}]$ ratio of $0.52 \pm$ 0.05 dex, which is significantly larger than in halo-, Carina-, and CEMP-stars at similar metallicity. These reference samples typically fall within \pm 0.2 dex of the Solar value. One exception is the CEMP- $s$ star S15-19 in the Sextans dSph (Honda et al. 2011), which, however, shows neutron-capture element patterns that are fully consistent with the AGB mass-transfer scenario, and no explanation of the high $\mathrm{Sc} / \mathrm{Fe}$ ratio was offered. Moreover, the horizontal branch star CS 29497-030 in the sample of Roederer et al. (2014), at the same [Fe/H], shares almost identical abundances of all the light elements in common, while its neutron-capture elements are highly enhanced, classifying that object as a CEMP$r / s$ star. A high Sc ratio could be indicative of a high-energy $\mathrm{SN}$ event and/or a high electron fraction in the nucleosynthetic environment (Tominaga et al. 2007). However, there is little evidence for this acting in the neutron-capture element patterns seen in ALW-8 (Sect. 4.8). Furthermore, the NLTE corrections for Sc in warmer stars are still largely unknown, hampering a detailed model comparison to pin down the origin of the Sc overenhancement (Hansen et al. 2011).

\subsection{Fe-peak elements: $\mathrm{Cr}, \mathrm{Mn}, \mathrm{Ni}$}

As the Fe-peak elements are co-produced with iron and their nucleosynthesis is decoupled from that in charge of the carbonenrichment and heavy element patterns in the CEMP stars, the $[\mathrm{Cr} / \mathrm{Fe}],[\mathrm{Mn} / \mathrm{Fe}]$, and $[\mathrm{Ni} / \mathrm{Fe}]$ ratios bear little surprise. ALW-8 is fully compatible with the (low-scatter) trends in the MW halo and those seen in other CEMP stars of either class, as well as with the abundances found in Carina stars, albeit their larger scatter. We did not attempt to correct our $\mathrm{Cr}$ and $\mathrm{Mn}$ abundances for NLTE effects, but we note that these would have no impact on the normality of ALW-8 in this abundance space.

\subsection{Neutron-capture elements: $Z n, Y, Z r, B a, E u$}

The $[\mathrm{Zn} / \mathrm{Fe}]$ ratio in ALW-8 and other CEMP stars is compatible with those in metal-poor halo stars, showing little scatter in the samples. Furthermore, there is no systematic difference to be seen between CEMP-no and $-s$ stars, which is to be expected as $\mathrm{Zn}$ is not significantly affected by $s$-process nucleosynthesis (Timmes et al. 1995; Kobayashi et al. 2006). However, C-normal stars in Carina show a large scatter towards intermediate metallicities, where their low-Zn and Fe-peak abundances indicate a lack of hypernovae, that is, energetic $\mathrm{SNe}$ that would lead to an overproduction of those elements (see also Hanke et al. 2017). Amongst the first-peak $n$-capture elements, only $\mathrm{Y}$ and $\mathrm{Zr}$ abundances could be determined. For Y, we employed the lines at 5200 and $5206 \AA$ (Fig. 5). While the latter feature is blended with a Cr I line, it can still be used for an abundance estimate, though with a larger error bar. As a result, we find an elevated [Y/Fe] ratio of $0.3 \mathrm{dex}$, which still overlaps with halo stars owing to the large scatter found in that MW component. We will further investigate this element in Sect. 5.2. Unblended $\mathrm{Zr}$ lines could not be detected in the spectrum and we placed an upper limit on the $\mathrm{Zr}$ abundance by synthesizing the lines at 6127 and $6134 \AA$, which are blended with CN bands; this yields an upper limit of $[\mathrm{Zr} / \mathrm{Fe}]<0.42$.

Barium is the only heavy neutron-capture element with several strong lines present in the spectrum. Four Ba II lines were detected in the spectrum of ALW-8 at 4934.1, 5853.7, 6141.7, and $6496.9 \AA$. However, the reddest of these lines is too blended 


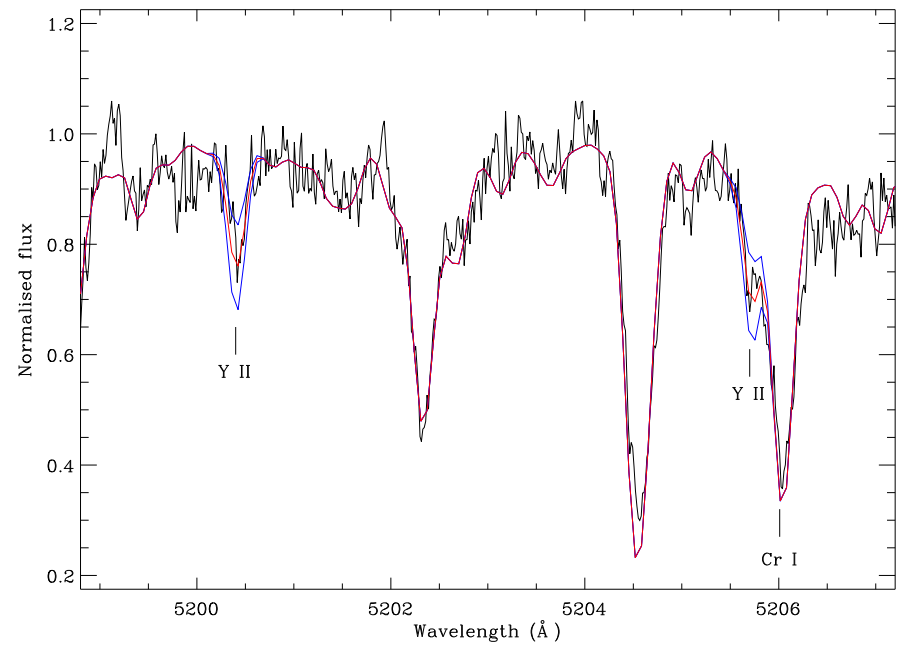

Fig. 5. Spectrum of ALW-8 around the Y II lines at $\lambda \lambda 5200$, $5206 \AA$ (black line), illustrating our spectral fitting. The best synthetic spectrum using $[\mathrm{Y} / \mathrm{Fe}]=0.26$ is plotted as a red line. The spectra shown in blue differ by \pm 0.3 from the best-fit value.

with molecular feature to be considered in our analysis. The remaining three lines yield a depleted value of $[\mathrm{Ba} / \mathrm{Fe}]=-0.55 \pm$ 0.20 that is in line with other metal-poor stars in Carina and the Galactic halo. The $[\mathrm{Ba} / \mathrm{Fe}]$ ratio shows a distinct bifurcation over a broad range in metallicity and both ALW stars in Carina studied by Abia et al. (2008) fall on the high-Ba band that is made up of CEMP- $s$ stars in binaries.

The weak Eu-line at $6645 \AA$ is blended with CN molecular features and we were only able to place an upper limit of $[\mathrm{Eu} / \mathrm{Fe}]<0.43$ from spectral synthesis.

\section{On the origin of elements}

Our abundance analysis of ALW-8 revealed that this CEMP star is enhanced in carbon as well as nitrogen. Considering its evolutionary phase, its ${ }^{12} \mathrm{C} /{ }^{13} \mathrm{C}$ ratio, and the depleted $\mathrm{Li}$ abundance give a sense that this star has undergone first dredge up (FDU). Nitrogen is the result of a $\mathrm{CN}$ cycle acting during the FDU. In this case, the original carbon in the star must have been even higher than what is measured now. Based on its low subsolar $[\mathrm{Ba} / \mathrm{Fe}]$ ratio and considering the lack of any strong $r$-process enhancement, ALW-8 can be unambiguously classified as a CEMP-no star (Beers \& Christlieb 2005). A mild enhancement is seen in the light neutron capture element $\mathrm{Y}$, which suggests the presence of a weak $r$-process activity. Thus, in the following, we consider the origin of the enhancements seen in different element tracers in ALW-8.

\subsection{Origin of carbon}

The strong C-enhancement of ALW-8 unambiguously confirms its nature as a CEMP star. ALW-8 is in the RGB phase where the primordial carbon abundance has been reduced due to the $\mathrm{CN}$ cycling and the presently observed abundance will be lower. While the moderate $[\mathrm{C} / \mathrm{N}]$ ratio of -0.2 dex alone would suggest that its surface composition has not been significantly altered by internal mixing during stellar evolution (Spite et al. 2005; Hansen et al. 2016a), other tracers of mixing paint a different picture. Amongst these are the strong depletion in lithium and the carbon isotopic ratio, where Spite et al. (2006) suggest an upper limit of ${ }^{12} \mathrm{C} /{ }^{13} \mathrm{C}<10$ as a proxy for effective deep mixing.
To further assess the impact of such processes, we consulted the corrections of Placco et al. (2014), which account for the evolutionary status of $\mathrm{C}$ - and N-rich stars. As these model calculations indicate, the surface composition of stars like ALW-8 has not been altered by more than $\sim 0.2$ over the course of its evolution. Since, during the hot $\mathrm{CNO}$ cycles, $\mathrm{N}$ is strongly enhanced at the expense of lowering the carbon abundance, the high $[\mathrm{C} / \mathrm{Fe}]$ we found in ALW-8 must have been a relic of external processes that enhanced the primordial gas to the observed high levels.

Carbon in CEMP-no stars can have several origins (we refer to, e.g., Skúladóttir et al. 2015, for a comprehensive overview). While the possibility of mass transfer from a AGB binary companion cannot be ruled out from a dynamical standpoint due to our single-epoch observations, the lack of significant $s$-process material renders this unlikely; in fact, the majority of CEMPno stars are not related to any binary mechanisms (Hansen et al. 2016b). The high value of carbon in CEMP-no stars can be the result of primordial, low- or zero-metallicity faint $\mathrm{SNe}$ that underwent mixing and fall-back mechanisms (Umeda \& Nomoto 2003). Alternative enrichers can be fast rotating massive stars (Meynet et al. 2006). However, both polluters produce excess abundances of $\mathrm{N}$ and $\mathrm{O}$ that are not produced to that extent in regular-energy SNe II. Indeed, our nitrogen and oxygen measurements indicate a strong enrichment in those elements, which, however, is more likely to be a consequence of the CNO cycling and mixing that the star experienced. Another piece of evidence against faint $\mathrm{SNe}$ being the sole producer of the high $\mathrm{C}$ is that they tend to have very low yields of the odd- $Z$ Fe-peak elements such as Mn, which shows a regular, non-depleted value in ALW-8.

The very similar abundance pattern of the CEMP-no star in the Sculptor dSph (Fig. 6) prompted Skúladóttir et al. (2015) to propose that a mix of faint and regular $\mathrm{SNe}$ II is required to reproduce the high $\mathrm{C}$ without invoking any of the peculiarities present in the faint $\mathrm{SNe}$ models. The regular pattern observed in the $\alpha$-elements and the high Na abundance seen in ALW-8 agrees with such a scenario and we conclude that Carina has also experienced some degree of enrichment by faint $\mathrm{SNe}$ that was, however, diluted with yields from SNe II. Overall, we note the very good agreement of the $[\alpha / \mathrm{Fe}]$ and Fe-peak abundance ratios between the Sculptor star and our Carina CEMP star (top panel of Fig. 6).

\subsection{Origin of neutron-capture elements}

At metallicities as low as ALW's $[\mathrm{Fe} / \mathrm{H}]$ of $-2.5 \mathrm{dex}$, the main source of the $n$-capture elements is $r$-process nucleosynthesis without major contributions from the $s$-process in AGB stars (Simmerer et al. 2004; Sneden et al. 2008). Generally, the ratio of first-to-second peak $n$-capture elements, such as [Y/Ba], is systematically lower in dSphs than in MW halo, owing to their low star forming efficiency (Fig. 14 in Tolstoy et al. 2009). The opposite is seen in ALW-8, which exhibits a high [Y/Ba] of 0.84 dex, which is otherwise seen in metal-poor halo stars below around -2.7 dex and also in the two most metal-poor Carina stars of Venn et al. (2012) as well as in two other dSph CEMP stars. This reflects significant departures from the abundance patterns that are governed by the main $r$-process in that the first-peak elements (such as Sr, Y, Zr) are systematically enhanced with respect to the heavier ones $(Z \gtrsim 56)$. Such trends prompted the need for an additional source of these elements via a weak $r$ - or weak $s$-process (e.g., Travaglio et al. 2004; Honda et al. 2006; Arcones \& Montes 2011). Also in ALW-8, only a low upper 


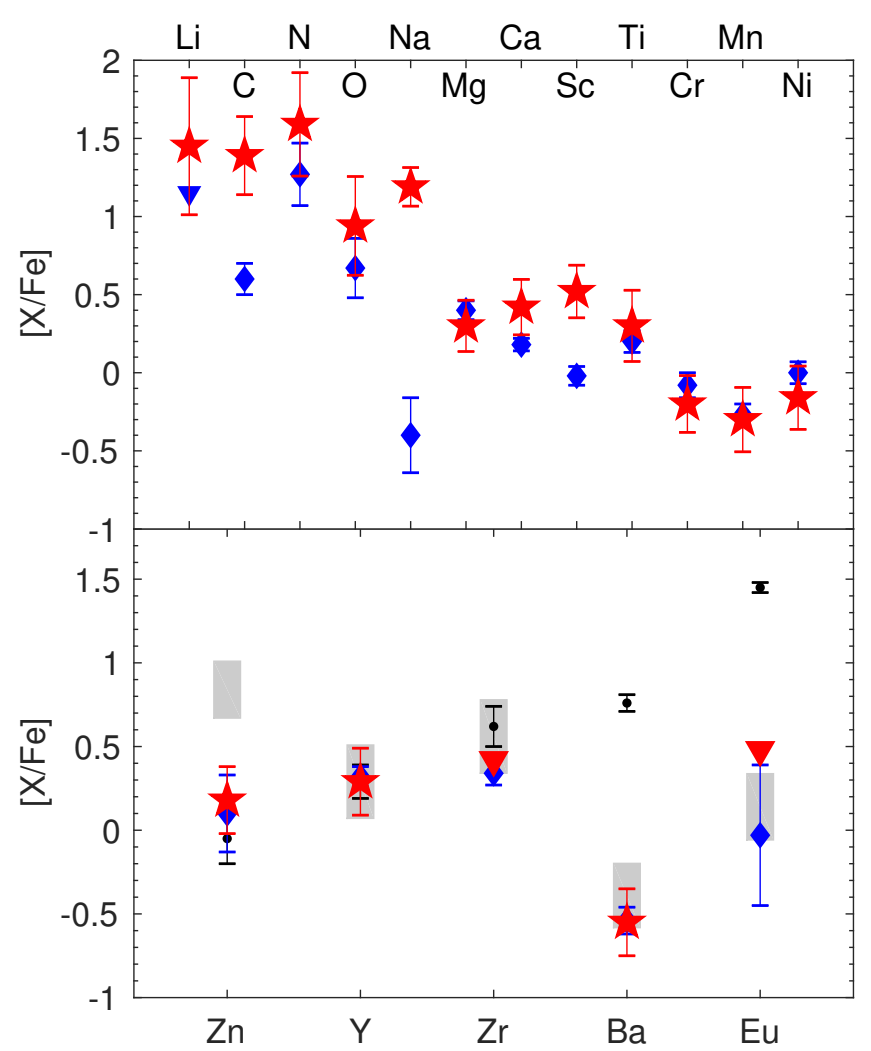

Fig. 6. Chemical element patterns for ALW-8 (red symbols) are shown for the lighter elements (top panel) and the neutron-capture element abundances (bottom panel). For comparison, we overplotted the CEMPno star in the Sculptor dSph (Skúladóttir et al. 2015, blue diamonds), and the weak and main $r$-process stars HD 122563 (Honda et al. 2006, gray shades) and CS 22892-052 (Sneden et al. 2003, black dots). Here, all stars were placed on the same Solar scale. All ratios for the heavier elements were normalized to $\mathrm{Y}$.

limit could be derived for $\mathrm{Eu}$, which serves as a tracer for main $r$-process.

Thus, in Fig. 6 (bottom panel), we place ALW-8 in the context of the $n$-capture processes by comparing the heavier element pattern to those in two metal-poor halo stars: HD 122563 $([\mathrm{Fe} / \mathrm{H}]=-2.82,[\mathrm{C} / \mathrm{Fe}]=-0.47$; Spite et al. 2005; Honda et al. 2006), which shows an archetypical weak $r$-process pattern, and CS 22892-052 $([\mathrm{Fe} / \mathrm{H}]=-3.12,[\mathrm{C} / \mathrm{Fe}]=0.89$; Sneden et al. 2003 , black dots) as a contender for enrichment via the main $r$-process. We note that all these reference stars have been normalized to the abundance of Y in ALW-8 in Fig. 6. It becomes immediately clear that the low $\mathrm{Ba}$ and $\mathrm{Eu}$ abundances are incompatible with an overproduction in the standard $r$-process. The scaled abundance ratio of $\mathrm{Zn}$ for HD 122563 deviates from the other stars shown here, while we note that the absolute $[\mathrm{Zn} / \mathrm{Fe}]$ ratios of these stars are consistent with one another. Thus, $\mathrm{Zn}$ appears comparably low; its origin rather lies in complete Si-burning with possible contributions from a weak $s$-process (Timmes et al. 1995; Travaglio et al. 2004; Venn et al. 2012). We note that our upper limit for $\mathrm{Zr}$ coincides with the lower error bound in HD 122563, while it should be produced in equal amounts to $\mathrm{Y}$ by the weak $r$-process. Ideally, an enhanced $\mathrm{Sr}$ abundance is expected from this process as well, but no $\mathrm{Sr}$ feature fell within our spectral range.

An alternative source of the excess in light $n$-capture elements is a weak $s$-process in fast rotating massive stars (Frischknecht et al. 2016). This scenario predicts an additional overproduction of $\mathrm{O}$ accompanying the enhancement in $\mathrm{C}, \mathrm{Sr}, \mathrm{Y}$, and $\mathrm{Zr}$. Indeed, the $[\mathrm{O} / \mathrm{Fe}]$ of 0.89 in $\mathrm{ALW}-8$ is high, and also for $\mathrm{HD} 122563$, a strongly elevated $[\mathrm{O} / \mathrm{Fe}]$ ratio between 0.6 and 1.1 dex has been reported (Barbuy et al. 2003; Afşar et al. 2016). Given the low number of elements that we measured in ALW-8 beyond $Z \gtrsim 30$, we cannot unambiguously conclude which of the processes ultimately led to the observed abundances and whether $\mathrm{O}$ was predominantly enhanced internally in the $\mathrm{CNO}$ processing or already imprinted in the gas alongside with the primordial carbon from earlier generations of faint $\mathrm{SNe}$ and/or fast rotators.

\section{Conclusions}

ALW-8 is the first CEMP-no star to be reported in the Carina $\mathrm{dSph}$ galaxy. The overall abundance pattern of the star suggests that the star was born from a medium that was enriched by a mixture of faint $\mathrm{SNe}$ and/or fast rotating massive stars, and lowmetallicity SNe II. The gas from which it formed had only undergone poor mixing. Moreover, the neutron-capture elements in ALW-8 are in excellent agreement with the only CEMP-no star in the Sculptor dSph and the weak- $r$ process star HD 122563. This suggests that, whichever process was responsible for the heavy element production must be an ubiquitous source to pollute the CEMP-no stars, acting independently of the environment, such as in the halo or in dSphs.

While a wealth of CEMP-no stars is known in the MW halo, the absolute number of such stars identified in nearby $\mathrm{dSph}$ and ultra-faint $\mathrm{dSph}$ satellites is low. Amongst these is the most iron-poor and most carbon-rich star in the Segue $1 \mathrm{dSph}$ $([\mathrm{Fe} / \mathrm{H}]=-3.5,[\mathrm{C} / \mathrm{Fe}]=2.3$; Norris et al. 2010b). While more iron-poor extragalactic stars exist in dSphs (Frebel et al. 2010a; Tafelmeyer et al. 2010), none of these are carbon-rich. On the other hand, the fraction of CEMP stars in the halo and in metalpoor dSph galaxies is known to significantly increase with decreasing metallicity, which has been interpreted as being due to the shift in the mean metallicity from lower to higher values as the luminosity of the galaxy increases (Salvadori et al. 2015).

Finally, the CEMP-no subclass is of particular interest as their fraction is largest in the outer Galactic halo, while CEMP- $s$ stars prevail in its inner, in-situ component (Carollo et al. 2014). This finding provides an immediate connection to the CEMP population in $\mathrm{dSphs}$ owing to the accretion origin of the outer halo (e.g., Searle \& Zinn 1978; Bullock \& Johnston 2005; Carollo et al. 2007; Cooper et al. 2013). As a consequence, the CEMP-no fraction in the Galactic satellites should be large and any additional candidate in this category is significant not only for nucleosynthetic considerations, but also for constraining the halo formation scenarios.

Acknowledgements. We thank M. Walker and T. T. Hansen for sharing their spectra of ALW-1 with us, and an anonymous referee for a helpful report. A.S. and A.K. acknowledge the Deutsche Forschungsgemeinschaft for funding from Emmy-Noether grant Ko 4161/1. This work used data obtained from the INSPECT database, version 1.0 (www . inspect-stars.net).

\section{References}

Abia, C., de Laverny, P., \& Wahlin, R. 2008, A\&A, 481, 161 Afşar, M., Sneden, C., Frebel, A., et al. 2016, ApJ, 819, 103 Aoki, W., Beers, T. C., Christlieb, N., et al. 2007, ApJ, 655, 492 Arcones, A., \& Montes, F. 2011, ApJ, 731, 5

Asplund, M., Grevesse, N., Sauval, A. J., \& Scott, P. 2009, ARA\&A, 47, 481 Azzopardi, M., Lequeux, J., \& Westerlund, B. E. 1986, A\&A, 161, 232 Barbuy, B., Meléndez, J., Spite, M., et al. 2003, ApJ, 588, 1072 Battaglia, G., Irwin, M., Tolstoy, E., et al. 2008, MNRAS, 383, 183 Battistini, C., \& Bensby, T. 2016, A\&A, 586, A49 
Beers, T. C., \& Christlieb, N. 2005, ARA\&A, 43, 531

Bensby, T., Feltzing, S., \& Oey, M. S. 2014, A\&A, 562, A71

Bonifacio, P., Caffau, E., Spite, M., et al. 2015, A\&A, 579, A28

Bullock, J. S., \& Johnston, K. V. 2005, ApJ, 635, 931

Carollo, D., Beers, T. C., Lee, Y. S., et al. 2007, Nature, 450, 1020

Carollo, D., Freeman, K., Beers, T. C., et al. 2014, ApJ, 788, 180

Cayrel de Strobel, G., \& Spite, M. 1988, The impact of very high S/N spectroscopy on stellar physics: proceedings of the 132nd Symposium of the International Astronomical Union held in Paris, France, June 29-July 3, 1987 IAU Symp., 132

Charbonnel, C., Brown, J. A., \& Wallerstein, G. 1998, A\&A, 332, 204

Cooper, A. P., D’Souza, R., Kauffmann, G., et al. 2013, MNRAS, 434, 3348

Cutri, R. M., Skrutskie, M. F., van Dyk, S., et al. 2003, VizieR Online Data Catalog: II/246

Fabrizio, M., Nonino, M., Bono, G., et al. 2015, A\&A, 580, A18

Frebel, A., Kirby, E. N., \& Simon, J. D. 2010a, Nature, 464, 72

Frebel, A., Simon, J. D., Geha, M., \& Willman, B. 2010b, ApJ, 708, 560

Frischknecht, U., Hirschi, R., Pignatari, M., et al. 2016, MNRAS, 456, 1803

Geisler, D., Smith, V. V., Wallerstein, G., Gonzalez, G., \& Charbonnel, C. 2005 AJ, 129, 1428

Geisler, D., Wallerstein, G., Smith, V. V., \& Casetti-Dinescu, D. I. 2007, PASP, 119, 939

Gratton, R. G., Sneden, C., Carretta, E., \& Bragaglia, A. 2000, A\&A, 354, 169

Grebel, E. K. 1997, in Rev. Mod. Astron., 10, 29

Hanke, M., Koch, A., Hansen, C. J., \& McWilliam, A. 2017, A\&A, 599, A97

Hansen, C. J., Nordström, B., Bonifacio, P., et al. 2011, A\&A, 527, A65

Hansen, C. J., Nordström, B., Hansen, T. T., et al. 2016a, A\&A, 588, A37

Hansen, T. T., Andersen, J., Nordström, B., et al. 2016b, A\&A, 586, A160

Hansen, T. T., Andersen, J., Nordström, B., et al. 2016c, A\&A, 588, A3

Honda, S., Aoki, W., Ishimaru, Y., Wanajo, S., \& Ryan, S. G. 2006, ApJ, 643, 1180

Honda, S., Aoki, W., Arimoto, N., \& Sadakane, K. 2011, PASJ, 63, 523

Idiart, T., \& Thévenin, F. 2000, ApJ, 541, 207

Ishigaki, M. N., Tominaga, N., Kobayashi, C., \& Nomoto, K. 2014, ApJ, 792 L32

Ji, A. P., Frebel, A., Ezzeddine, R., \& Casey, A. R. 2016a, ApJ, 832, L3

Ji, A. P., Frebel, A., Simon, J. D., \& Chiti, A. 2016b, ApJ, 830, 93

Kirby, E. N., Lanfranchi, G. A., Simon, J. D., Cohen, J. G., \& Guhathakurta, P. 2011, ApJ, 727, 78

Kobayashi, C., Umeda, H., Nomoto, K., Tominaga, N., \& Ohkubo, T. 2006, ApJ, 653,1145

Kobayashi, C., Tominaga, N., \& Nomoto, K. 2011, ApJ, 730, L14

Koch, A., \& Edvardsson, B. 2002, A\&A, 381, 500

Koch, A., Grebel, E. K., Wyse, R. F. G., et al. 2006, AJ, 131, 895

Koch, A., Grebel, E. K., Gilmore, G. F., et al. 2008, AJ, 135, 1580

Koch, A., McWilliam, A., Preston, G. W., \& Thompson, I. B. 2016, A\&A, 587, A124

Kupka, F., Piskunov, N., Ryabchikova, T. A., Stempels, H. C., \& Weiss, W. W. 1999, A\&AS, 138, 119

Lai, D. K., Lee, Y. S., Bolte, M., et al. 2011, ApJ, 738, 51
Lee, Y. S., Beers, T. C., Kim, Y. K., et al. 2017, ApJ, 836, 91 Lemasle, B., Hill, V., Tolstoy, E., et al. 2012, A\&A, 538, A100 Lind, K., Primas, F., Charbonnel, C., Grundahl, F., \& Asplund, M. 2009, A\&A, 503,545

Lind, K., Asplund, M., Barklem, P. S., \& Belyaev, A. K. 2011, A\&A, 528, A103

Masseron, T., Johnson, J. A., Plez, B., et al. 2010, A\&A, 509, A93

Masseron, T., Johnson, J. A., Lucatello, S., et al. 2012, ApJ, 751, 14

Mészáros, S., Allende Prieto, C., Edvardsson, B., et al. 2012, AJ, 144, 120

Meynet, G., Ekström, S., \& Maeder, A. 2006, A\&A, 447, 623

Monelli, M., Pulone, L., Corsi, C. E., et al. 2003, AJ, 126, 218

Mould, J., \& Aaronson, M. 1983, ApJ, 273, 530

Nonino, M., Bertin, E., da Costa, L., et al. 1999, A\&AS, 137, 51

Norris, J. E., Gilmore, G., Wyse, R. F. G., Yong, D., \& Frebel, A. 2010a, ApJ, 722, L104

Norris, J. E., Wyse, R. F. G., Gilmore, G., et al. 2010b, ApJ, 723, 1632

Placco, V. M., Frebel, A., Beers, T. C., \& Stancliffe, R. J. 2014, ApJ, 797, 21

Plez, B. 2012, Astrophysics Source Code Library [record ascl: 1205.004]

Plez, B., \& Cohen, J. G. 2005, A\&A, 434, 1117

Roederer, I. U., Preston, G. W., Thompson, I. B., et al. 2014, AJ, 147, 136

Salgado, C., Da Costa, G. S., Yong, D., \& Norris, J. E. 2016, MNRAS, 463, 598

Salvadori, S., Skúladóttir, Á., \& Tolstoy, E. 2015, MNRAS, 454, 1320

Sbordone, L., Bonifacio, P., Caffau, E., et al. 2010, A\&A, 522, A26

Schlegel, D. J., Finkbeiner, D. P., \& Davis, M. 1998, ApJ, 500, 525

Searle, L., \& Zinn, R. 1978, ApJ, 225, 357

Shetrone, M., Venn, K. A., Tolstoy, E., et al. 2003, AJ, 125, 684

Simmerer, J., Sneden, C., Cowan, J. J., et al. 2004, ApJ, 617, 1091

Sivarani, T., Beers, T. C., Bonifacio, P., et al. 2006, A\&A, 459, 125

Skúladóttir, Á., Tolstoy, E., Salvadori, S., et al. 2015, A\&A, 574, A129

Smecker-Hane, T. A., Stetson, P. B., Hesser, J. E., \& Lehnert, M. D. 1994, AJ, 108,507

Sneden, C., Cowan, J. J., Lawler, J. E., et al. 2003, ApJ, 591, 936

Sneden, C., Cowan, J. J., \& Gallino, R. 2008, ARA\&A, 46, 241

Spite, M., \& Spite, F. 1982, Nature, 297, 483

Spite, M., Cayrel, R., Plez, B., et al. 2005, A\&A, 430, 655

Spite, M., Cayrel, R., Hill, V., et al. 2006, A\&A, 455, 291

Starkenburg, E., Shetrone, M. D., McConnachie, A. W., \& Venn, K. A. 2014, MNRAS, 441, 1217

Tafelmeyer, M., Jablonka, P., Hill, V., et al. 2010, A\&A, 524, A58

Thompson, I. B., Ivans, I. I., Bisterzo, S., et al. 2008, ApJ, 677, 556

Timmes, F. X., Woosley, S. E., \& Weaver, T. A. 1995, ApJS, 98, 617

Tolstoy, E., Venn, K. A., Shetrone, M., et al. 2003, AJ, 125, 707

Tolstoy, E., Hill, V., \& Tosi, M. 2009, ARA\&A, 47, 371

Tominaga, N., Umeda, H., \& Nomoto, K. 2007, ApJ, 660, 516

Travaglio, C., Gallino, R., Arnone, E., et al. 2004, ApJ, 601, 864

Umeda, H., \& Nomoto, K. 2003, Nature, 422, 871

Venn, K. A., Shetrone, M. D., Irwin, M. J., et al. 2012, ApJ, 751, 102

Walker, M. G., Mateo, M., \& Olszewski, E. W. 2009, AJ, 137, 3100

Yoon, J., Beers, T. C., Placco, V. M., et al. 2016, ApJ, 833, 20 


\section{Appendix A: Additional table}

Table A.1. Line list for the EW analysis, abundance and abundance uncertainties to $1 \sigma$ scatter in the stellar parameters for ALW-8.

\begin{tabular}{|c|c|c|c|c|c|c|c|c|}
\hline Element & $\begin{array}{l}\lambda \\
\AA\end{array}$ & $\begin{array}{c}\chi \\
(\mathrm{eV})\end{array}$ & $\log (g f)$ & $\begin{array}{l}E W \\
\mathrm{~m} \AA\end{array}$ & $A(x)$ & $\begin{array}{c}\delta T \\
\pm 100 \mathrm{~K}\end{array}$ & $\begin{array}{c}\delta \log g \\
\pm 0.25 \mathrm{dex}\end{array}$ & $\begin{array}{c}\delta \xi \\
\pm 0.2 \mathrm{~km} \mathrm{~s}^{-1}\end{array}$ \\
\hline $\mathrm{Li} I$ & 6707.70 & 0.00 & 0.17 & syn & 0.00 & \pm 0.30 & $\mp 0.10$ & \pm 0.01 \\
\hline O I & 6300.31 & 0.00 & -9.82 & syn & 6.93 & $\mp 0.10$ & $\mp 0.03$ & \pm 0.01 \\
\hline O I & 6363.78 & 0.02 & -10.30 & syn & 7.23 & $\mp 0.10$ & $\mp 0.03$ & \pm 0.01 \\
\hline $\mathrm{Na} \mathrm{I}$ & 5682.63 & 2.09 & -0.70 & 58 & 4.84 & $\mp 0.03$ & \pm 0.07 & $\mp 0.02$ \\
\hline $\mathrm{Na} I$ & 5688.21 & 2.09 & -0.45 & 82 & 4.91 & $\mp 0.02$ & \pm 0.09 & $\mp 0.03$ \\
\hline Mg I & 5528.40 & 4.34 & -0.62 & 120 & 5.57 & $\mp 0.00$ & \pm 0.11 & $\mp 0.06$ \\
\hline $\mathrm{Ca} \mathrm{I}$ & 5261.70 & 2.52 & -0.59 & 67 & 4.51 & $\mp 0.04$ & \pm 0.08 & $\mp 0.04$ \\
\hline $\mathrm{Ca} \mathrm{I}$ & 5588.75 & 2.53 & 0.31 & 100 & 4.07 & $\mp 0.02$ & \pm 0.10 & $\mp 0.07$ \\
\hline $\mathrm{Ca} \mathrm{I}$ & 5594.46 & 2.52 & 0.05 & 102 & 4.36 & $\mp 0.02$ & \pm 0.10 & $\mp 0.08$ \\
\hline Ca I & 6102.72 & 1.88 & -0.86 & 85 & 4.05 & $\mp 0.06$ & \pm 0.09 & $\mp 0.04$ \\
\hline $\mathrm{Ca} \mathrm{I}$ & 6122.22 & 1.89 & -0.38 & 167 & 4.66 & $\mp 0.05$ & \pm 0.15 & $\mp 0.09$ \\
\hline Sc II & 5526.79 & 1.77 & 0.02 & 100 & 1.27 & \pm 0.00 & $\mp 0.13$ & $\mp 0.08$ \\
\hline Sc II & 5657.90 & 1.51 & -0.60 & 70 & 1.08 & $\mp 0.02$ & $\mp 0.16$ & $\mp 0.04$ \\
\hline Sc II & 5684.20 & 1.51 & -1.07 & 55 & 1.15 & $\mp 0.03$ & $\mp 0.16$ & $\mp 0.02$ \\
\hline Ti I & 4981.73 & 0.85 & 0.50 & 180 & 3.26 & $\mp 0.11$ & \pm 0.14 & $\mp 0.15$ \\
\hline Ti I & 4999.50 & 0.83 & 0.25 & 95 & 2.14 & $\mp 0.11$ & \pm 0.10 & $\mp 0.07$ \\
\hline Ti I & 5020.03 & 0.84 & -0.41 & 84 & 2.66 & $\mp 0.12$ & \pm 0.10 & $\mp 0.05$ \\
\hline Ti I & 5024.84 & 0.82 & -0.60 & 47 & 2.30 & $\mp 0.14$ & \pm 0.08 & $\mp 0.02$ \\
\hline Ti I & 5036.46 & 1.44 & 0.13 & 60 & 2.63 & $\mp 0.11$ & \pm 0.09 & $\mp 0.03$ \\
\hline Ti I & 5173.74 & 0.00 & -1.11 & 135 & 2.83 & $\mp 0.15$ & \pm 0.08 & $\mp 0.12$ \\
\hline Ti I & 5210.38 & 0.05 & -0.88 & 120 & 2.44 & $\mp 0.15$ & \pm 0.09 & $\mp 0.09$ \\
\hline Ti II & 4865.61 & 1.12 & -2.67 & 51 & 2.78 & $\mp 0.04$ & $\mp 0.15$ & $\mp 0.02$ \\
\hline Ti II & 5336.77 & 1.58 & -1.63 & 97 & 3.01 & $\mp 0.00$ & $\mp 0.14$ & $\mp 0.08$ \\
\hline Ti II & 6491.56 & 2.05 & -1.79 & 33 & 2.74 & $\mp 0.02$ & $\mp 0.17$ & $\mp 0.01$ \\
\hline $\mathrm{Cr} \mathrm{I}$ & 5247.57 & 0.96 & -1.64 & 66 & 2.99 & $\mp 0.12$ & \pm 0.09 & $\mp 0.03$ \\
\hline $\mathrm{Cr} \mathrm{I}$ & 5296.69 & 0.98 & -1.40 & 95 & 3.18 & $\mp 0.11$ & \pm 0.10 & $\mp 0.07$ \\
\hline $\mathrm{Cr} \mathrm{I}$ & 5345.80 & 1.00 & -0.98 & 139 & 3.44 & $\mp 0.10$ & \pm 0.11 & $\mp 0.12$ \\
\hline $\mathrm{Cr} \mathrm{I}$ & 5348.31 & 1.00 & -1.29 & 64 & 2.66 & $\mp 0.12$ & \pm 0.09 & $\mp 0.03$ \\
\hline $\mathrm{Cr} \mathrm{I}$ & 5409.77 & 1.03 & -0.72 & 120 & 2.91 & $\mp 0.10$ & \pm 0.10 & $\mp 0.10$ \\
\hline Mn I & 5394.68 & 0.00 & -3.50 & 70 & 2.61 & $\mp 0.18$ & \pm 0.06 & $\mp 0.03$ \\
\hline Mn I & 5432.55 & 0.00 & -3.79 & 54 & 2.70 & $\mp 0.18$ & \pm 0.06 & $\mp 0.02$ \\
\hline Fe I & 4891.49 & 2.85 & -0.11 & 160 & 4.81 & $\mp 0.04$ & \pm 0.14 & $\mp 0.11$ \\
\hline Fe I & 4966.09 & 3.32 & -0.87 & 94 & 5.22 & $\mp 0.04$ & \pm 0.07 & $\mp 0.08$ \\
\hline Fe I & 5001.86 & 3.88 & 0.01 & 88 & 5.01 & $\mp 0.02$ & \pm 0.06 & $\mp 0.07$ \\
\hline $\mathrm{Fe} \mathrm{I}$ & 5194.94 & 1.55 & -2.08 & 156 & 4.90 & $\mp 0.09$ & \pm 0.06 & $\mp 0.16$ \\
\hline $\mathrm{Fe} \mathrm{I}$ & 5195.47 & 4.21 & -0.08 & 57 & 5.05 & $\mp 0.04$ & \pm 0.04 & $\mp 0.03$ \\
\hline Fe I & 5215.18 & 3.27 & -0.87 & 100 & 5.17 & $\mp 0.04$ & \pm 0.07 & $\mp 0.08$ \\
\hline $\mathrm{Fe} \mathrm{I}$ & 5225.53 & 0.11 & -4.78 & 128 & 4.97 & $\mp 0.15$ & \pm 0.01 & $\mp 0.12$ \\
\hline Fe I & 5242.49 & 3.63 & -0.96 & 50 & 5.04 & $\mp 0.06$ & \pm 0.03 & $\mp 0.02$ \\
\hline $\mathrm{Fe} \mathrm{I}$ & 5307.36 & 1.61 & -2.98 & 93 & 4.84 & $\mp 0.11$ & \pm 0.04 & $\mp 0.07$ \\
\hline Fe I & 5586.76 & 3.36 & -0.12 & 133 & 4.97 & $\mp 0.03$ & \pm 0.09 & $\mp 0.10$ \\
\hline Fe I & 6546.24 & 2.76 & -1.53 & 103 & 4.97 & $\mp 0.08$ & \pm 0.02 & $\mp 0.07$ \\
\hline Fe II & 5197.58 & 3.23 & -2.09 & 55 & 5.03 & \pm 0.05 & $\mp 0.18$ & $\mp 0.03$ \\
\hline Fe II & 5234.63 & 3.22 & -2.23 & 45 & 4.96 & \pm 0.04 & $\mp 0.18$ & $\mp 0.02$ \\
\hline $\mathrm{Ni} \mathrm{I}$ & 4980.17 & 3.60 & 0.00 & 95 & 4.62 & $\mp 0.01$ & \pm 0.04 & $\mp 0.08$ \\
\hline $\mathrm{Ni} \mathrm{I}$ & 5424.64 & 1.95 & -2.77 & 85 & 4.84 & $\mp 0.08$ & \pm 0.00 & $\mp 0.06$ \\
\hline $\mathrm{Ni} \mathrm{I}$ & 6108.11 & 1.67 & -2.45 & 52 & 3.59 & $\mp 0.11$ & $\mp 0.01$ & $\mp 0.02$ \\
\hline $\mathrm{Ni} \mathrm{I}$ & 6314.65 & 1.92 & -1.77 & 62 & 3.39 & $\mp 0.10$ & $\mp 0.01$ & $\mp 0.03$ \\
\hline $\mathrm{Ni} \mathrm{I}$ & 6643.63 & 1.67 & -2.30 & 75 & 3.69 & $\mp 0.11$ & $\mp 0.01$ & $\mp 0.04$ \\
\hline $\mathrm{Zn} \mathrm{I}$ & 4810.53 & 4.07 & -0.13 & 30 & 2.19 & \pm 0.02 & $\mp 0.12$ & $\mp 0.01$ \\
\hline Y II & 5200.41 & 0.99 & -0.57 & syn & 0.00 & \pm 0.05 & \pm 0.10 & \pm 0.01 \\
\hline Y II & 5205.73 & 1.03 & -0.34 & syn & 0.00 & \pm 0.05 & \pm 0.10 & \pm 0.01 \\
\hline $\mathrm{Zr}$ II & 6127.48 & 0.15 & -1.06 & syn & $<0.50$ & \pm 0.30 & $\mp 0.30$ & \pm 0.01 \\
\hline Zr II & 6134.59 & 0.00 & -1.28 & syn & $<0.50$ & \pm 0.30 & $\mp 0.30$ & \pm 0.01 \\
\hline $\mathrm{Ba}$ II & 4934.08 & 0.00 & -0.15 & syn & -1.20 & \pm 0.10 & \pm 0.10 & $\mp 0.01$ \\
\hline Ba II & 5853.68 & 0.60 & -0.91 & syn & -0.50 & \pm 0.15 & \pm 0.20 & $\mp 0.10$ \\
\hline Ba II & 6141.73 & 0.70 & -0.03 & syn & -0.90 & \pm 0.10 & \pm 0.10 & $\mp 0.10$ \\
\hline Eu II & 6645.09 & 1.38 & 0.12 & syn & $<-1.50$ & \pm 0.70 & \pm 0.50 & \pm 0.01 \\
\hline
\end{tabular}

